\title{
Análisis comparativo entre cooperativas de ahorro y crédito y bancos en el Ecuador
}

\author{
Comparative analysis between credit union and banks in Ecuador
}

\author{
Jorge Cárdenas Muñoz' (D) (8), Eduardo Treviño Saldívar² (D) (8), Gina Cuadrado Sánchez ${ }^{3}$ (D) (8i), \\ Janice Ordóñez Parra ${ }^{4}$ (D) (8)
}

\author{
Cómo citar \\ Cárdenas Muñoz, J., Treviño Saldívar. E., Cuadrado Sánchez, G. y Ordóñez Parra, J. (2021). Análisis comparativo entre \\ cooperativas de ahorro y crédito y bancos en el Ecuador. Socialium, 5(2), 159-184. \\ https://doi.org/10.26490/uncp.sl.2021.5.2.1000
}

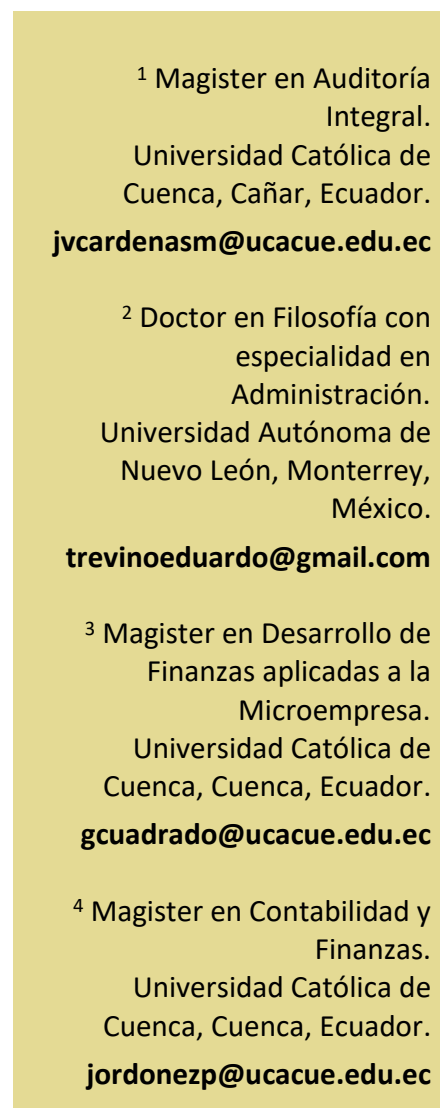

Arbitrado por pares ciegos Recibido: 20/12/2020 Aceptado: $16 / 01 / 2021$

\section{RESUMEN}

El sistema financiero constituye un pilar fundamental en la economía de un país, donde las cooperativas y los bancos son los actores que sobresalen en cuanto a cobertura y servicios. El objetivo es establecer las particularidades en el funcionamiento de los sectores cooperativo y bancario en el Ecuador. La metodología empleada en el desarrollo de este trabajo es cualitativa, su alcance es descriptivo-transversal y de carácter documental, para lo cual, se utilizaron fuentes secundarias, mediante una revisión bibliográfica a profundidad de una serie de estadísticas con corte transversal al 2020, emitidas por la Asociación de Bancos Privados del Ecuador y la Superintendencia de Economía Popular y Solidaria y Superintendencia de Bancos. Los resultados presentan las particularidades en el funcionamiento de los sectores cooperativo y bancario en el Ecuador, analizando su marco regulatorio, constitución, principios fundacionales, filosofía, modelo de negocio, gobernanza, proceso de intermediación financiera, evolución y participación en el mercado, principales indicadores financieros y contribución a la economía real. Se puede concluir que existen características diferenciadoras profundas desde los aspectos filosófico y social entre estos dos representantes del sistema financiero nacional, destacándose que las Cooperativas de Ahorro y Crédito tienen una fuerte presencia en el ámbito social regida por principios y valores; en tanto que el sector bancario prevalece como número uno en cuanto a cobertura y movilización de capitales.

Palabras clave: Cooperativas de ahorro y crédito; bancos; economía social; caracterización; sistema financiero.

\section{ABSTRACT}

The financial system constitutes a fundamental pillar in the economy of a country, where cooperatives and banks are the actors that stand out in terms of coverage and services. The objective is to establish the particularities in the operation of the cooperative and banking sectors in Ecuador. The methodology used in the development of this work is qualitative, its scope is descriptive-transversal and of a documentary nature, for which, secondary sources were used, through an in-depth bibliographic review of a series of statistics with a cross-section to 2020, issued by the Association of Private Banks of Ecuador and the Superintendency of Popular and Solidarity Economy and Superintendency of Banks. The results present the particularities in the operation of the cooperative and banking sectors in Ecuador, analyzing their regulatory framework, constitution, founding principles, philosophy, business model, governance, financial intermediation process, evolution and participation in the market, main indicators financial and contribution to the real economy. It can be concluded that there are profound differentiating characteristics from the philosophical and social aspects between these two representatives of the national financial system, highlighting that the Savings and Credit Cooperatives have a strong presence in the social sphere governed by principles and values; while the banking sector prevails as number one in terms of coverage and capital mobilization.

Keywords: Credit unions; banks; social economy; characterization; financial system. 


\section{Introducción}

En los últimos tiempos el crecimiento del sistema financiero ha implicado una mayor especialización de las actividades que realizan, Terrones (2013) afirma que las instituciones financieras (IFIS) se han alejado del modelo de banca tradicional, que consiste en la intermediación de fondos entre ahorristas y tomadores de créditos, para enfocarse en la provisión de productos y servicios especializados que demandan una infraestructura financiera particular.

Aunque, los bancos y cooperativas ofrecen los mismos servicios como cuentas de ahorro, préstamos, captación, pagos de varios servicios, estas entidades tienen objetivos diferentes. Es necesario distinguir que los bancos comerciales son sociedades con fines de lucro orientadas a obtener utilidades, en donde los accionistas o inversores son los propietarios del banco. Por otro lado, las cooperativas de ahorro y crédito son de propiedad de los socios que utilizan sus servicios, son organizaciones sin fines de lucro y sus principios se basan en la cooperación para obtener beneficios comunes.

Ahora bien, el sistema financiero del siglo XXI demanda exigencias adicionales, ya que se enfoca, además de la intermediación financiera clásica de captar ahorro y colocar crédito, en proveer una variedad de productos y servicios que permitan realizar transacciones ágiles y eficientes enfocándose en altos estándares de calidad y servicio. Martínez, Rivas, Rositas, Treviño (2015) sostienen:

El acceso a otros mercados económicos y financieros, ha obligado a las instituciones financieras y a las empresas, a tener empleados mejor preparados y con habilidades y competencias que les permitan analizar, desde un entorno macro y microeconómico, el comportamiento de la economía y así, poder tomar decisiones rápidas y acertadas buscando disminuir los posibles efectos adversos, los cuales pudieran ocasionar pérdidas económicas a las instituciones. (p. 1133)

Así también, en el año 2015 se oficializaron las nuevas reglas de mercado a partir de la entrada en vigencia del Código Orgánico Monetario y Financiero (COMYF) (Asamblea Nacional del Ecuador, 2014), en este contexto, las entidades financieras tienen el desafío de consolidar nuevas estrategias para el desarrollo de las actividades financieras de acuerdo a lo establecido en el COMYF del 12 de septiembre del 2014 En su Art. 143 que manifiesta:

Actividad financiera es el conjunto de operaciones y servicios que se efectúan entre oferentes, demandantes y usuarios, para facilitar la circulación de dinero y realizar intermediación financiera; tienen entre sus finalidades preservar los depósitos y atender los requerimientos de financiamiento para la consecución de los objetivos de desarrollo del país. (p. 45)

Además, la Superintendencia de Bancos y Seguros del Ecuador en su página web define a un Sistema 
financiero como;

El conjunto de instituciones que tiene como objetivo canalizar el ahorro de las personas. Esta canalización de recursos permite el desarrollo de la actividad económica (producir y consumir) haciendo que los fondos lleguen desde las personas que tienen recursos monetarios excedentes hacia las personas que necesitan estos recursos. Los intermediarios financieros crediticios se encargan de captar depósitos del público y, por otro, prestarlo a los demandantes de recursos (Superintendencia de Bancos, 2020).

Sin embargo, tanto para las entidades del sistema financiero privado, público y de economía popular y solidaria, existe el arbitraje regulatorio por parte de los entes de control pertinentes, cuyas atribuciones son: velar por la seguridad, estabilidad, transparencia y solidez del sistema financiero ecuatoriano y sobre todo proteger los intereses de sus clientes y socios. Es por ello, que el objetivo del artículo es establecer las particularidades en el funcionamiento de los sectores cooperativo y bancario en el Ecuador, colocando especial atención en su marco regulatorio, forma de constitución, principios fundacionales, filosofía, modelo de negocio, gobernanza, proceso de intermediación financiera, evolución y participación en el mercado.

\section{Método}

Tipo y diseño de estudio. Se utilizó un diseño investigativo no experimental con metodología cualitativa, transversal y documental, caracterizada porque los datos no serán alterados por los investigadores, sino se estudiarán tal como se obtenga la información. El enfoque cualitativo posibilita conocer la conceptualización, características diferenciadoras y evolución del sistema financiero ecuatoriano representado por los bancos y cooperativas.

Población y muestra. Para la investigación se utilizo la pesquisa de información documental y bibliográfica de los reportes estadísticos de las instituciones estatales reguladoras de la banca ecuatoriana al 2020.

Instrumentos de recolección de datos. Para la investigación se utilizó fuentes secundarias emitidas por la Asociación de Bancos Privados del Ecuador (ASOBANCA); y los organismos de control: la Superintendencia de Economía Popular y Solidaria (SEPS) y Superintendencia de Bancos. Al respecto Muñoz (2011), establece que en estas investigaciones tanto la recopilación de información, como el análisis, comprobaciones, aplicaciones, prácticas, conocimientos y métodos utilizados para obtener conclusiones 
se realiza en el medio en el que se presenta el fenómeno o hecho de estudio pues se analizarán los datos en un único momento del tiempo

Procedimientos de la recolección de datos. La recolección de datos se efectuó utilizando fuentes secundarias, mediante una revisión bibliográfica a profundidad de una serie de estadísticas con corte transversal al 2020 de la ASOBANCA, SEPs y Superintendencia de Bancos.

Aspectos éticos. En la investigación los aspectos éticos responden a la responsabilidad y los principios fundamentales por tratar con estadísticas oficiales de una sociedad democrática, al servicio del gobierno, la economía y el público con datos sobre la situación económica, demográfica, social y ambiental, compiladas y puestas a disposición de manera imparcial propiciando el debate.

Análisis de datos. A partir de la caracterización de los datos estadísticos se procedió a su comparación, análisis y obtención de resultados que permiten determinar las singularidades de las cooperativas de ahorro y crédito y de la banca utilizando hojas de cálculo de Microsoft Excel para tabular los datos.

\section{Resultado}

\section{Referencias teóricas sobre el sistema financiero}

El sistema financiero ecuatoriano está compuesto por: Bancos, Cooperativas de Ahorro y Crédito, Sociedades Financieras, Mutualistas, Instituciones financieras públicas y Compañías de Seguros como se puede observar en la figura 1.

\section{Figura 1}

Sistema Financiero Ecuatoriano

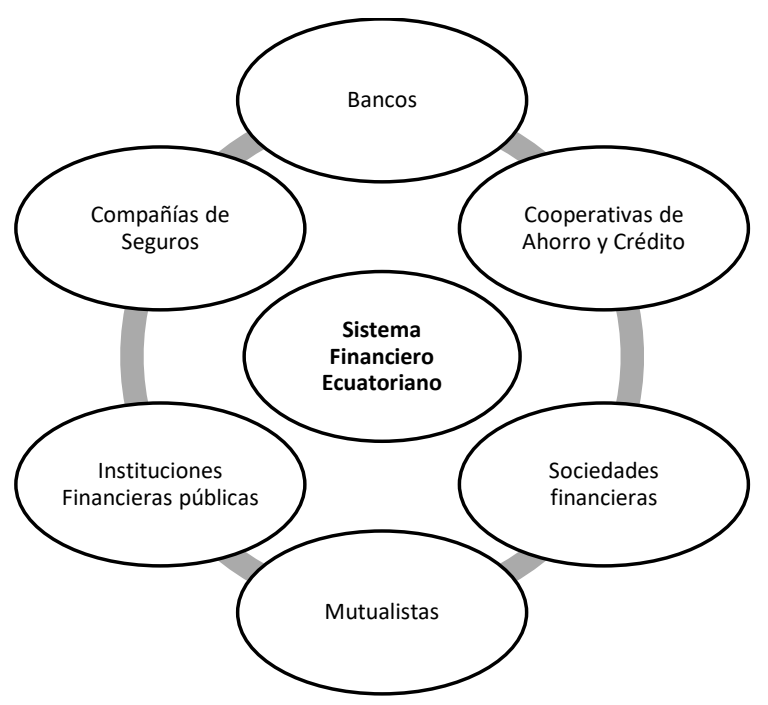


El sistema financiero está regulado por cuatro cuerpos legales: Constitución de la República del Ecuador, Código Orgánico Monetario Financiero (COMYF), la Ley Orgánica de Instituciones del Sistema Financiero; y la Ley Orgánica de Economía Popular y Solidaria (LOEPS). Así mismo los organismos de control son la Superintendencia de Bancos; y la Superintendencia de Economía Popular y Solidaria.

Las instituciones que cuentan con mayor cobertura en el sistema financiero ecuatoriano son los Bancos privados y las Cooperativas de Ahorro y Crédito (COACs) y, razón por la cual este estudio se focalizará en estos dos sectores.

Las COACs surgen como un movimiento de Economía Popular y Solidaria o Economía Social que propende el desarrollo y crecimiento de un territorio en base a la generación de empleo, distribución equitativa de excedentes, que, a decir de Castelló y Trías (2015) combina rentabilidad, inclusión social y gestión democrática.

Las cooperativas constituyen un sujeto jurídico diferenciado del conjunto de sociedades mercantiles, que presentan rasgos sustantivos derivados, entre otros, de la existencia de una regulación legal propia, con una larga tradición y; en las variadas circunstancias políticas, económicas y sociales han tenido que hacer frente demostrado su capacidad generadora de bienestar y riqueza para sus socios, constituyéndose las cooperativas en el motor de la vida económica y social en numerosos territorios y el principal instrumento empresarial al servicio de sus habitantes (Segui et al.,, 2011).

Según Turner (1996), una cooperativa de ahorro y crédito es una entidad de autoayuda financiera a sus socios para ahorrar dinero y los recursos agrupados se utilizan para proporcionar préstamos a bajo costo, opera dentro de un área de ubicación claramente definida y con un vínculo común entre sus miembros. Los objetivos de una cooperativa de ahorro y crédito son múltiples y podrían clasificarse como una combinación de fines sociales Turner (1996) los resumió en:

- Promover el ahorro

- Proporcionar préstamos a los miembros a una tasa de interés razonable.

- Ayudar a los miembros a hacer un uso efectivo de sus recursos financieros.

Por ley, los miembros de las cooperativas de ahorro y crédito deben estar unidos por un "vínculo común de ocupación o asociación o pertenecer a grupos dentro de un vecindario, comunidad o distrito" (Hannan, 2002 , p. 1). En las cooperativas de ahorro y crédito se pueden observar tendencias y problemas de desarrollo similares. Éstas incluyen:

- Una primera etapa en la que, las cooperativas de ahorro y crédito comienzan como instituciones locales que operan en una comunidad determinada o radio, cuya membresía se otorga en base a un vínculo 
común, en la cual los miembros ofrecen servicios voluntarios y están muy motivados, factores que la hacen altamente competitiva (Ofei, 2001).

- Una etapa posterior es la interacción entre cooperativas de crédito primarias. Esta es la base para desarrollar el crédito secundario o terciario. A través de la creación de un fondo central y la cooperación con otros para fortalecer el desarrollo.

McKillop et ál. (2020) también ha relatado sobre el desarrollo de la industria de cooperativas de ahorro y crédito en tres etapas que las denomina "naciente", "transición" y finalmente a una etapa "madura". La industria naciente representa una etapa de desarrollo donde las cooperativas de crédito son vistas como organizaciones de autoayuda con un fuerte propósito social. Los atributos clave de esta etapa incluyen un tamaño de activo pequeño, un vínculo común estrecho entre miembros y énfasis en el voluntarismo. La etapa de transición en la que se siembran las semillas del cambio dentro de las cooperativas de crédito, muestra una relajación de la filosofía y los ideales cooperativos. Esto es causado por la necesidad de lograr eficiencia de costos y economías de escala debido al crecimiento de los activos y la competencia en el mercado.

La etapa madura pone fin a la trayectoria de desarrollo de las cooperativas de crédito. De acuerdo con McKillop et al. (2020) los atributos clave de esta etapa son la adopción de nuevas características como: gran tamaño de activos, una interpretación menos restrictiva del requisito de fianza común para los miembros, cambio en sus estrategias operativas, la diversificación de los productos y servicios, profesionalidad de gestión en lugar de voluntarismo, transformación de su naturaleza ideológica e institucional. Las cooperativas de ahorro y crédito se vuelven más orientadas a los negocios y los bonos comunes han perdido protagonismo.

En sus intentos de competir eficazmente en sus entornos, se ha convertido en obligatorio para las cooperativas de ahorro y crédito que asuman las características de las instituciones financieras convencionales (Akenten et al.,, 2009).

Según López (2011) En la Antigua Grecia durante el imperio Romano prestamistas aceptaban depósitos y prestaban dinero. Los judíos realizaban sus transacciones de préstamos en una mesa con un mantel verde con sillas. El nombre "BANCO" deriva de la palabra italiana banco, que significa "escritorio". Cuando se generaba grandes pleitos por el cobro abusivo de los intereses, algunos deudores inconformes, de furia rompían los bancos. Entonces se decía que ese judío estaba en "bancarrota" (banca-rota), de ahí el origen de esas palabras que se utilizan en la actualidad. Otros autores como Pérez (2011) sostiene:

La expresión banco tiene su origen en los empréstitos públicos contraídos por las ciudades italianas al comienzo del siglo XII. En efecto, los empréstitos públicos fueron denominados Monti 
en Italia, que significa fondo común. En aquel entonces los germanos, que tenían una gran influencia en Italia comenzaron a designar el fondo común, constituido por las contribuciones de varias personas, con la expresión Bank, juntamente con el equivalente vocablo Monte. Al poco tiempo Bank fue italianizado en Banco y la acumulación de empréstitos públicos fue llamada indiferentemente Monte o Banco. (p. 60)

De acuerdo a la revista ABC del Banco Central del Ecuador (2012) establece:

Es una institución cuya función es captar recursos (dinero) del público a través de depósitos, para luego destinarlos a los sectores que lo requieran (como las empresas) bajo la forma de préstamos o créditos. Los bancos son conocidos como intermediarios de recursos, puesto que los captan de unos sectores y los entregan a otros que los necesitan. (p. 31)

Las entidades financieras constituyen un elemento clave para el desarrollo socio-económico de un país. Se encargan de fomentar el ahorro de un segmento de la población que dispone de excedentes de recursos, y canalizarlos a créditos e inversiones. Las operaciones de crédito permiten a las personas y empresas el desarrollo de actividades productivas y de servicios. Los recursos de los ahorristas tienen rendimientos que incentivan el crecimiento de captaciones de depósitos. De esta manera el objetivo del sistema financiero determinado en el COMYF del 12 de septiembre del 2014, Sección 3 De las finalidades y objetivos, Art. 402 establece:

Las entidades financieras que conforma el sector financiero, tendrán como finalidad y objetivo el ejercicio de actividades financieras, las cuales podrán ejercerlas, previa autorización del Estado, de acuerdo con este Código, preservando los depósitos y atendiendo los requerimientos de intermediación financiera de la ciudadanía. (Asamblea Nacional Constituyente, 2008, p. 118)

Respecto al origen de los bancos, desde la existencia del hombre como un ser social que trabaja y adquiere alimentos y bienes para sobrevivir, ha habido trueque y el intercambio de monedas según corresponde a cada época. Una breve reseña histórica nos da a conocer que en Asiria y Babilonia aproximadamente en el año 2000 AC inicia como un primer prototipo de banco de comerciantes del mundo antiguo a través de préstamos de granos a los agricultores y negociantes. Hernández (2017) sostiene:

De acuerdo a los historiadores, el primer banco moderno fue el Banco di San Giorgio, fundado en Génova, Italia, en el año 1406. La originalidad de las operaciones bancarias de los italianos fue rápidamente copiada por los países del norte de Europa, principalmente los holandeses, los ingleses y los suecos. (p.6)

Entre el siglo XII y XIV los bancos conocieron un renacimiento importante, se le denomina la Banca Clásica, ya que los hombres de negocios de Italia del norte desarrollaron notablemente las operaciones de cambio. 
No solamente fueron expertos manipuladores de piezas metálicas, sino también, mediante una letra de cambio, podían acreditar a una persona, en una fecha determinada, en moneda nacional o extranjera, ante un determinado corresponsal. Así, muchos comerciantes recurrían a los bancos para tratar sus negocios con terceros. Éstos, por una comisión, los representaban o se comprometían por ellos, con lo que eran comerciantes y banqueros al mismo tiempo (Hernández, 2017).

En el siglo XIX, los bancos conocen una época de crecimiento y estabilidad, marcada por el desarrollo de los institutos de emisión, la multiplicación de las casas de "alta banca", que actúan como consejeros, corredores o mandatarios en grandes operaciones financieras; la creación de los grandes bancos comerciales, cuyo capital estaba altamente distribuido en el público, y el nacimiento de las instituciones parabancarias, destinadas a responder a las necesidades específicas de la clientela, denominándola como la Banca moderna especializada (Romero, 2017).

A mediados del siglo XX se le conoce como la Expansión Bancaria. Carvajal, A., y Zuleta, H. (1997) por cuanto con el desarrollo de la era del consumo de masas, el crecimiento de las transacciones comerciales y financiera desembocó en los primero movimientos liberalizadores de los sistemas bancarios, integrados por diferentes componentes, y existe una fuerte competencia de productos y servicios no sólo bancos del estado o privados, sino también aquellos que pertenecen a colectividades locales o regionales, a sindicatos o cooperativas.

Finalmente, la época de la Globalización bancaria a finales del siglo XX, "cuya tendencia es un mercado universal, con una revolución en el modo de hacer banca con nuevas estrategias, diversificación, diseños organizativos más eficaces, fusiones, alianzas, mayor orientación y beneficios para el cliente" (Hernández, 2017, p.7).

De acuerdo a lo antes expuesto la Banca busca mantener un sistema solvente, seguro, moderno y competitivo que esté en condiciones de incrementar sus vínculos con los diversos sectores productivos y apoyar el proceso económico y social a nivel nacional. Otro aspecto fundamental es que la sociedad confíe nuevamente en las instituciones financieras y se pueda reactivar de mejor manera la economía en el país. “dejando atrás un tiempo donde la crisis estaba presente en todas las economías, ... generando un gran desaliento entre la población" (Tristán et al., 2019, p. 82). De igual manera es importante el ofertar productos y servicios innovadores gracias a los avances tecnológicos que facilitarán las actividades financieras de sus usuarios.

Los resultados del estudio se presentarán en dos partes como lo sugieren McKillop et al. (2020), la primera considerando las características estructurales y de comportamiento de los dos tipos de instituciones 
financieras, para en una segunda parte dar paso a la presentación sobre la evolución, rendimiento y contribución a la economía real.

\section{Primera parte: Características estructurales y de comportamiento}

\section{Las cooperativas de ahorro y crédito en el Ecuador}

El sector financiero popular y solidario en el Ecuador comprende las cooperativas de ahorro y crédito, cajas de ahorro, bancos comunales y mutualistas. Estas organizaciones se identifican por su lógica asociativa y realizan actividades de intermediación financiera y responsabilidad social con sus socios situando las finanzas al servicio del ser humano (figura 2).

\section{Figura 2}

Economía popular y solidaria

\section{Economía popular y solidaria}

\begin{tabular}{|c|c|c|c|c|c|c|}
\hline \multicolumn{2}{|c|}{ Sector no financiero } & \multicolumn{5}{|c|}{ Sector financiero } \\
\hline $\begin{array}{l}\text { Organizaciones } \\
\text { comunitarias, } \\
\text { asociativas y } \\
\text { cooperativistas }\end{array}$ & $\begin{array}{l}\text { Uunidades } \\
\text { económicas } \\
\text { populares }\end{array}$ & $\begin{array}{l}\text { Cooperativas de } \\
\text { ahorro y crédito }\end{array}$ & Mutualistas & Cajas centrales & $\begin{array}{l}\text { Cajas solidarias y } \\
\text { de ahorro }\end{array}$ & $\begin{array}{l}\text { Cajas y bancos } \\
\text { comunales. }\end{array}$ \\
\hline
\end{tabular}

Según el COMYF en su artículo 445 indica que las cooperativas de ahorro y crédito son organizaciones formadas por personas naturales o jurídicas que se unen voluntariamente bajo los principios establecidos en la Ley Orgánica de Economía Popular y Solidaria (LOEPS), con el objetivo de realizar actividades de intermediación financiera y de responsabilidad social con sus socios. (Asamblea Nacional de la República del Ecuador, 2014).

La LOEPS en su artículo 21 define al sector cooperativo como sociedades de personas que se han unido en forma voluntaria para satisfacer sus necesidades económicas, sociales y culturales en común, mediante una empresa de propiedad conjunta y de gestión democrática, con responsabilidad jurídica de derecho privado e interés social (SEPS, 2018).

Las cooperativas de ahorro y crédito en el Ecuador están clasificadas en cinco segmentos de acuerdo al valor de sus activos conforme se establece en la siguiente tabla 1. 
Tabla 1

Segmentos de las cooperativas de ahorro y crédito

\begin{tabular}{ll}
\hline Segmento & \multicolumn{1}{c}{ Activos } \\
\hline Segmento 1 & Mayor a $80.000 .000,00$ \\
Segmento 2 & Mayor a 20.000.000,00 hasta $80.000 .000,00$ \\
Segmento 3 & Mayor a 5.000.000,00 hasta 20.000.000,00 \\
Segmento 4 & Mayor a 1.000.000,00 hasta 5.000.000,00 \\
Segmento 5 & Hasta un 1.000.000,00 \\
& Cajas de ahorro, bancos comunales y cajas comunales
\end{tabular}

Nota. Elaborado a partir de las normas para la segmentación de las entidades del sector financiero popular y solidario (2020).

En el primer segmento están las 31 instituciones más grandes (activos superiores a USD 80 millones); en el último nivel se encuentran 277 cajas de ahorro, bancos y cajas comunales. Según el Informe de Rendición de Cuentas 2019 de la Superintendencia de Economía Popular y Solidaria (2019) se manifiesta que a diciembre de 2019 el Sector Financiero Popular y Solidario (SFPS):

(...) se encuentra integrado por 547 cooperativas de ahorro y crédito, una caja central, cuatro asociaciones mutualistas de ahorro y crédito para la vivienda, y una corporación. Las 552 entidades que conforman el sector registran 7,79 millones de certificados de aportación, con una suma de USD 16132 millones en activos, USD 13784 millones en pasivos y USD 2345 millones en patrimonio (..) El SFPS representa aproximadamente un tercio de la actividad de intermediación financiera del país, al concentrar el $27,2 \%$ de las captaciones y el $28,6 \%$ de las colocaciones del sistema financiero nacional privado. Sin embargo, al interior del sector, se observa gran heterogeneidad entre las instituciones que lo conforman; así, el 6,7\% de las entidades (segmento 1 y mutualistas 2) concentran el $64,0 \%$ de los certificados de aportación, el 78,9\% del activo, 79,7\% del pasivo y $73,9 \%$ del patrimonio total del sector. (p. 5,6$)$

Tabla 2

Composición del sector financiero popular y solidario por segmentos a diciembre del 2019

\begin{tabular}{lccccc}
\hline Segmento & Nro. Entidades & $\begin{array}{c}\text { Nro. Certificados de } \\
\text { Aportación }\end{array}$ & $\begin{array}{c}\text { Activos } \\
\text { (USD Millones) }\end{array}$ & $\begin{array}{c}\text { Cartera de Crédito } \\
\text { (USD Millones) }\end{array}$ & $\begin{array}{c}\text { Depósitos } \\
\text { (USD Millones) }\end{array}$ \\
\hline Segmento 1 & 33 & 4.973 .502 & 11.671 & 8.595 & 9.233 \\
Mutualistas & 4 & 16.206 & 1.051 & 681 & 895 \\
Segmento 2 & 42 & 1.383 .119 & 1.893 & 7.534 & 1.349 \\
Segmento 3 & 81 & 797.714 & 958 & 364 & 694 \\
Segmento 4 & 166 & 475.266 & 461 & 76 & 320 \\
Segmento 5 & 226 & 152.238 & 98 & 12.015 & 64 \\
Total & 552 & 7.798 .045 & 16.132 & 12.555 \\
\hline
\end{tabular}

Nota. Elaborado a partir de Superintendencia de Economía Popular y Solidaria SEPS (2019). 


\section{Ranking de las cooperativas de ahorro y crédito}

El movimiento cooperativo ecuatoriano mantiene una fuerte presencia a nivel latinoamericano y del Caribe, situándose en el segundo lugar con una cobertura del $19,38 \%$ y el $20.65 \%$ del sistema financiero nacional al 2018 y 2019 respectivamente de participación dentro del sistema financiero ecuatoriano con un nivel de activos con $\$ 58.682$ millones en el 2018 y 62.111 millones en el 2019, colocándolo en el octavo puesto entre los sistemas financieros de América Latina y el Caribe (DGRV, 2019).

Conforme esta segmentación las cooperativas ecuatorianas que lideran el sector se ubican en el segmento 1 destacándose la presencia de: Cooperativa Juventud Ecuatoriana Progresista, Cooperativa Jardín Azuayo y la Cooperativa de la Policía Nacional, las cuales concentran un 27,2\% del volumen de crédito por el sistema cooperativo.

A continuación, se observa las posiciones en los rankings de acuerdo a la presencia en el sistema financiero nacional, las cooperativas más grandes y con mayor participación individual en América Latina y el Caribe (tabla 3).

Tabla 3

Ranking Cooperativas Ecuatorianas

\begin{tabular}{lccc}
\hline \multicolumn{1}{c}{ Cooperativa } & Ranking Nacional & Ranking Latinoamérica y el Caribe & TOP 10 \\
\hline Juventud Ecuatoriana Progresista & 1 & 3 & 1 \\
Jardín Azuayo & 2 & 11 & 8 \\
Policía Nacional & 3 & 14 & 9 \\
\hline
\end{tabular}

Nota. Datos y Ranking de Cooperativas de Ahorro y Crédito en América Latina y el Caribe 2018 (DGRV, 2019).

\section{La Banca Ecuatoriana}

El sector financiero ecuatoriano conformado por la Banca atravesó por diferentes sucesos que afectaron a la economía del país, sin embargo, lo que se busca es proveer de una mayor y mejor variedad de productos y servicios que permitan realizar transacciones ágiles y eficientes enfocándose en altos estándares de calidad, en atención a los parámetros establecidos en el COMYF.

Por su parte el Banco Central del Ecuador (2021) constituye un elemento indispensable en el flujo de efectivo, facilitando las actividades económicas de la población y los sectores productivos a través de las siguientes funciones:

- Posibilita que las persona dispongan de billetes y monedas en la cantidad, calidad y en las denominaciones necesarias. 
- Facilita los pagos y cobros que todas las personas realizan en efectivo, o a través del sistema financiero privado.

- Evalúa, monitorea y controla permanentemente la cantidad de dinero de la economía, para lo que utiliza como herramienta el encaje bancario.

- Revisa la integridad, transparencia y seguridad de los recursos del Estado que se manejan a través de nuestras operaciones bancarias.

- Ofrece a las personas, empresas y autoridades pública información para la toma de decisiones financieras y económica.

En este contexto las IFIS registradas en la Superintendencia de Bancos, con corte al 2020 son 32 instituciones financieras correspondientes a la Banca Privada y Pública.

\section{Tabla 4}

Banca Ecuatoriana a Julio 2020

\begin{tabular}{|c|c|c|}
\hline Instituciones & Cantidad & Porcentaje \\
\hline Banca Privada & 25 & $78 \%$ \\
\hline Banca Pública & 7 & $22 \%$ \\
\hline Total & 32 & $100 \%$ \\
\hline
\end{tabular}

\section{Ranking del Sistema de Bancos Privados}

La Superintendencia de Bancos a través de su Dirección de Estudios y Gestión de la Información, presenta información con corte mensual y anual de la situación financiera de la banca privada. A continuación, se muestra un resumen de las seis entidades que lideran el sector en base a su posición financiera en cuanto a sus activos, patrimonio y resultados.

\section{Tabla 5}

Ranking en base a la participación de las Principales Cuentas del Balance al 31 de julio del 2020 (en miles de dólares y porcentajes)

\begin{tabular}{lccc}
\hline \multicolumn{1}{c}{ Banco } & Activos & Patrimonio & Resultados \\
\hline Pichincha & 11.577 .325 & 1.246 .012 & 35.286 \\
Pacifico & 6.224 .952 & 769.897 & 18.475 \\
Guayaquil & 5.184 .355 & 502.127 & 12.310 \\
Produbanco & 5.034 .143 & 425087 & 15.203 \\
Internacional & 3.710 .410 & 357921 & 19.551 \\
Bolivariano & 3.618 .074 & 328240 & 16.690
\end{tabular}

Nota. Elaborado a partir de Superintendencia de Bancos (2020). 


\section{Comparativo de características estructurales y de comportamiento}

Uno de los principales resultados de este estudio es establecer con claridad las singularidades de las cooperativas de ahorro y crédito y los bancos, entre las cuales sobresalen: la legislación que la regula, su forma de constitución, gobierno, principios y valores que se detallan en la siguiente tabla:

\section{Tabla 6}

Características Cooperativas y Bancos

\begin{tabular}{|c|c|}
\hline Cooperativa & Banco Tradicional \\
\hline $\begin{array}{l}\text { - Regulada por la Ley Orgánica de Economía Popular } \\
\text { y Solidaria }\end{array}$ & $\begin{array}{l}\text { - } \quad \text { Regulado por la Ley Orgánica del Sistema } \\
\text { Financiero Nacional }\end{array}$ \\
\hline $\begin{array}{l}\text { - Controlada por la Superintendencia de Economía } \\
\text { Popular y Solidaria }\end{array}$ & - Controlado por la Superintendencia de Bancos \\
\hline - Sociedad de personas & - $\quad$ Sociedad de capitales \\
\hline - Membresía abierta y voluntaria & - $\quad$ Directorio cerrado \\
\hline - Democracia: una persona un voto & - Democracia de capitales: una acción un voto \\
\hline - Fin: servicio, buen vivir, desarrollo & - Su fin es la ganancia y la acumulación \\
\hline $\begin{array}{l}\text { - Financia a emprendimientos sociales y } \\
\text { ambientalmente sostenibles }\end{array}$ & $\begin{array}{l}\text { - Financia emprendimientos económicamente } \\
\text { rentables }\end{array}$ \\
\hline - Vela por el interés común & - $\quad$ Cuida el interés individual \\
\hline - Intercooperación e intracooperación & - $\quad$ Competencia de libre mercado \\
\hline - Dirección participativa y horizontal & - Dirección centralizada y vertical \\
\hline $\begin{array}{l}\text { - Propiedad colectiva: los factores de la producción } \\
\text { pertenecen a sus miembros }\end{array}$ & $\begin{array}{l}\text { - Propiedad individual: en función del porcentaje de } \\
\text { capital }\end{array}$ \\
\hline - Conformada por socios & - Conformada por accionistas \\
\hline - Atiende a socios & - $\quad$ Atiende a clientes \\
\hline $\begin{array}{l}\text { - Genera excedentes, ahorro, aumento de } \\
\text { patrimonio }\end{array}$ & - $\quad$ Genera utilidades \\
\hline $\begin{array}{l}\text { - Distribución equitativa de excedentes en forma de } \\
\text { beneficios: balance social }\end{array}$ & - $\quad$ Reparto de utilidades en base al aporte de capital \\
\hline - Control social, autogestión, corresponsabilidad & - Control particular, gestión, responsabilidad \\
\hline - Trabajo sobre el capital & - $\quad$ Capital sobre el trabajo \\
\hline - Educación y formación en valores y principios & - Capacitación: planes de carrera \\
\hline - Identidad, pertenece a un territorio & - $\quad$ No tiene pertenencia a un territorio \\
\hline $\begin{array}{l}\text { - Compromiso con la comunidad y transformación } \\
\text { social }\end{array}$ & $\begin{array}{l}\text { - Filantropía y responsabilidad social corporativa } \\
\text { como estrategia organizacional }\end{array}$ \\
\hline - Consumo ético & - $\quad$ Prevalece el consumismo \\
\hline $\begin{array}{l}\text { - Transparencia en la información y rendición de } \\
\text { cuentas }\end{array}$ & - Información restringida al directorio \\
\hline
\end{tabular}

Nota: Elaboración propia en base a (UNAE, 2019; McKillopet al., 2020; Superintendencia de Economía Popular y Solidaria, 2019).

La caracterización presentada muestra que el cooperativismo financiero parte de un modelo de negocios en el que prevalece el ser humano sobre el capital, primando el beneficio colectivo sobre el individual, en el cual sus miembros se asocian en base a un sueño común en búsqueda de dar solución a una 
problemática social abordada en base a principios fundamentales en los que se ejerce con democracia, transparencia y equidad.

En tanto que la banca se rige por la libre competencia de mercado en la cual se busca la generación de utilidades para sus accionistas, basado en un sistema capitalista en el cual la propiedad, las decisiones, la gestión entre otros, se centra en la proporción de su capital. Sin embargo, es innegable que el crédito bancario ha sido por años un elemento clave para el financiamiento de nuevos productos de inversión, así como para el crecimiento de la capacidad instalada de las actividades productivas que incentivan a nuevas fuentes de trabajo en todos los sectores. Cumpliendo así con su rol de financiar el desarrollo productivo del país.

\section{Segunda parte: evolución en cifras, rendimiento y contribución a la economía}

\section{Evolución del cooperativismo}

La evolución del cooperativismo en el Ecuador muestra una particular importancia, pasando de 26 cooperativas que manejaban 75 millones de dólares de activos, con una participación del $0.8 \%$ en el sistema financiero nacional en el año 1998; a 603 cooperativas que movilizan 12.823 millones de dólares de activos y concentran el 19,7\% de participación en el sistema financiero ecuatoriano al 2019 (DGRV, 2019).

Hasta antes del 2012 había más de 1000 cooperativas dispersas, se implementaron controles más rigurosos, por lo que el número se redujo. En ese proceso, varias COAC cerraron por una mala administración, liquidez e incumplimiento de normativa, registrando 608 organizaciones a diciembre del 2018 (El Comercio, 2019). Las COACs más grandes del país, alrededor de 40, eran controladas por la Superintendencia de Bancos y el resto estaba bajo supervisión del Ministerio de Inclusión Económica y Social, para el 2012 pasaron a ser supervisadas por la Superintendencia de Economía Popular y Solidaria. En los últimos ocho años, las cooperativas de ahorro y crédito (COACs) dieron un salto importante, mientras en el 2012 sus activos alcanzaron USD 6.027 millones, a junio 2020 sumaron 13.703 millones, según datos la Superintendencia de la Economía Popular y Solidaria (SEPS, 2018, ASOBANCA, 2020). Ese mismo comportamiento también se observa en indicadores como los pasivos, el patrimonio, los créditos, los depósitos, las utilidades y el número de socios. Para el 2012, estas entidades colocaron USD 4.562 millones en préstamos a sus socios y a junio 2020 la cifra llegó a 10.143 millones, con un 42\% de cobertura (ASOBANCA, 2020).

La expansión del sector cooperativo se explica, entre otros factores, porque las cooperativas tienen mayor presencia en poblaciones rurales, sus productos y servicios se han diversificado y colocan más del 
$60 \%$ del microcrédito en el país. Por cada dólar que capta en las zonas rurales, el sector financiero popular y solidario coloca USD 1,66. En los lugares con mayor índice de pobreza, la colocación es de USD 1,71. Como resultado de lo anterior, el número de socios (clientes) aumentó.

La cartera de crédito vencida registrada al cierre del año 2019 en el sector cooperativo mantiene una tendencia a crecer (Asobanca, 2020). Lo cual es un factor que afecta a las instituciones financieras y en parte se debe a la situación económica que se vive en el país lo cual no permite cumplir con las cuotas establecidas y en otros casos se registra como incobrables para las instituciones financieras.

\section{Figura 3}

Cartera Cooperativas de Ahorro y Crédito

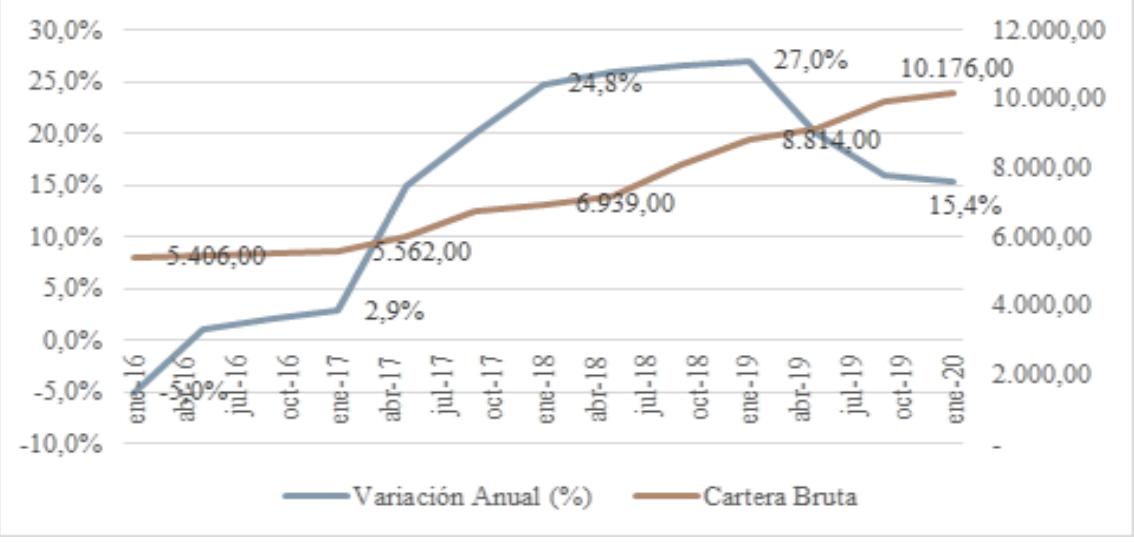

En el sector de la Cooperativas de ahorro y crédito es mucho más notoria la tendencia a la baja a inicios del año 2019 en sus captaciones, en primera instancia debido a la difícil situación que se enfrenta en la actualidad en el tema laboral, incremento en los precios, lo cual no permite a los usuarios el fomentar el ahorro.

\section{Figura 4}

Captaciones Cooperativas de Ahorro y Crédito

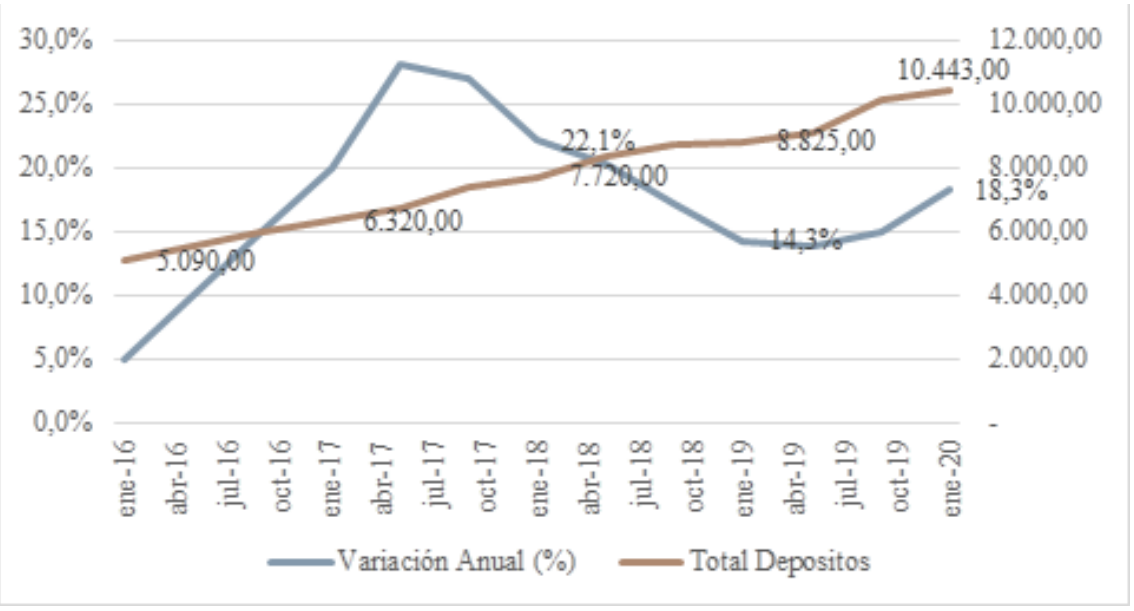


Al finalizar el mes de junio del 2020, se puede visualizar que el total de activos de las Cooperativas de los segmentos 1 y 2 (son los segmentos que agrupan a las cooperativas de ahorro y crédito más grandes del país) registran un crecimiento del 1,9\% con relación al mes de mayo. Lo propio se registró en sus pasivos de manera mensual con un aumento del 1,9\%. En lo relacionado al saldo de sus activos se ubicó en USD 13.703 millones y sus pasivos en USD 11.656 millones lo cual representa un crecimiento anual del $13,6 \%$ y $13,7 \%$ respectivamente. De su parte el patrimonio contabilizó un saldo de USD 1.991 millones lo que significó una variación anual de 15,5\%. En lo concerniente a la cuenta de ingresos registró una variación anual de 10,8\%, en tanto que la cuenta de gastos registró un incremento en $16,4 \%$. Para finalizar la utilidad establecida fue de USD 55 millones, frente a las USD 86 millones de junio del 2019.

\section{Tabla 7}

Principales cuentas Cooperativas a junio 2019 - junio 2020

\begin{tabular}{lcccccc}
\hline & jun-19 & dic-19 & may-20 & jun-20 & $\begin{array}{c}\text { Variación } \\
\text { Mensual }\end{array}$ & $\begin{array}{c}\text { Variación } \\
\text { anual }\end{array}$ \\
\hline Total, activos & 12.065 & 13.275 & 13.448 & 13.703 & $1,9 \%$ & $13,6 \%$ \\
Contingentes & 196 & 204 & 232 & 231 & $-0,3 \%$ & $18,2 \%$ \\
Total, Pasivos & 10.255 & 11.329 & 11.440 & 11.656 & $1,9 \%$ & $13,7 \%$ \\
Patrimonio & 1.724 & 1.946 & 1.967 & 1.991 & $1,2 \%$ & $15,5 \%$ \\
Ingresos & 804 & 1.698 & 732 & 891 & & $10,8 \%$ \\
Gastos & 78 & 1.552 & 691 & 835 & & $16,4 \%$ \\
Utilidad Neta & 86 & 146 & 41 & 55 & & $-35,9 \%$ \\
\hline
\end{tabular}

\section{Evolución de La Banca}

El desarrollo de la banca ecuatoriana es muy reciente, puesto que la historia reseña que los primeros bancos se establecieron a fines del siglo XIX. Precisamente en Guayaquil, ciudad pujante, dinámica y comercial por excelencia, se creó y estableció el primer banco emisor en 1859 con el nombre de "Banco Particular de Manuel Antonio de Luzarraga", en febrero de 1861 se realizó la primera emisión de billetes. Posteriormente el 5 de noviembre de 1868 se firmó la escritura de constitución del "Banco del Ecuador". El 14 de diciembre de 1869 inicia sus operaciones el "Banco Quito". Una vez más Guayaquil con su ancestral vocación comercial y su gente emprendedora funda el "Banco de Crédito Hipotecario" en 1871. Más tarde en 1885 se organizó en Guayaquil el “Banco Anglo Ecuatoriano” y el Banco Territorial en 1886 (Romero, 2017). 
Según Espinosa (2000) En 1998 bajo la administración de Jamil Mahuad Witt como presidente de Ecuador, la banca soportó la peor crisis financiera de la historia de este país, en el año 1999 dio como resultado la quiebra de varios de los más grandes bancos de la ciudad de Guayaquil, como el Banco del Progreso, La Previsora y, el entonces mayor banco de Ecuador, Filanbanco lo que representó \$8.600 millones de pérdidas para el país. Superado esos sucesos, por su parte de acuerdo a los datos de la Superintendencia de Bancos (2010), la Banca pasó de una colocación de 70.000 operaciones y USD 455 millones en el 2006, a 504.000 operaciones (crecimiento del 620\%) y USD 1.743 millones (crecimiento del 283\%) en el 2010.

Según la última información establecida por la Asobanca (2020) la relación entre patrimonio técnico constituido y total de activos ponderados por riesgos, al cierre de mayo 2020 , llego a $13,9 \%$ superando el requerimiento legal del 9\%, siendo su crecimiento anual desde el año 2016 en 0,6 puntos porcentuales lo cual evidencia la solvencia que tiene hasta la actualidad la Banca Ecuatoriana. De igual manera los depósitos totales crecieron en USD 372 millones con relación al mes anterior, cerrando a junio de 2020 en USD. 33.527 millones lo cual representa un crecimiento del 6,1\% con respecto al año anterior.

Por sucesión, los principales bancos según su actual solvencia, manejo de activos, patrimonio y resultados de acuerdo al reporte de la Superintendencia de Bancos (2020) son: Banco del Pichincha con USD 11.577 millones, Banco del Pacífico USD 6.224 millones, Banco de Guayaquil USD 5.184 millones Promerica USD 5.034 millones, Banco Internacional USD 3.710 millones, y Banco Bolivariano USD 3.618 millones. Actualmente están los organismos de control velando por el cumplimiento de las actividades por parte de las IFIS, de igual manera existen varios reportes que se pueden visualizar en las páginas oficiales, auditoras calificadas, y revistas económicas de amplio reconocimiento que dan a conocer el ranking de las mejores IFIS, de manera anual.

En las Instituciones financieras el proceso de intermediación financiera es un pilar fundamental y la razón por la cual el sistema financiero aporta en la economía de un país, en los últimos años en lo referente a las colocaciones, la cartera de crédito en la Banca privada tiene un comportamiento similar al del sector de Cooperativas de ahorro y crédito, la cartera de crédito vencida tiene una tendencia a crecer lo cual es un factor que afecta al sistema financiero y como se mencionó anteriormente en parte se debe a la situación económica del país, lo cual no permite cumplir con las cuotas establecidas y en otros casos se registra como cuentas incobrables para los bancos. 


\section{Figura 5}

Cartera Bancos Privados

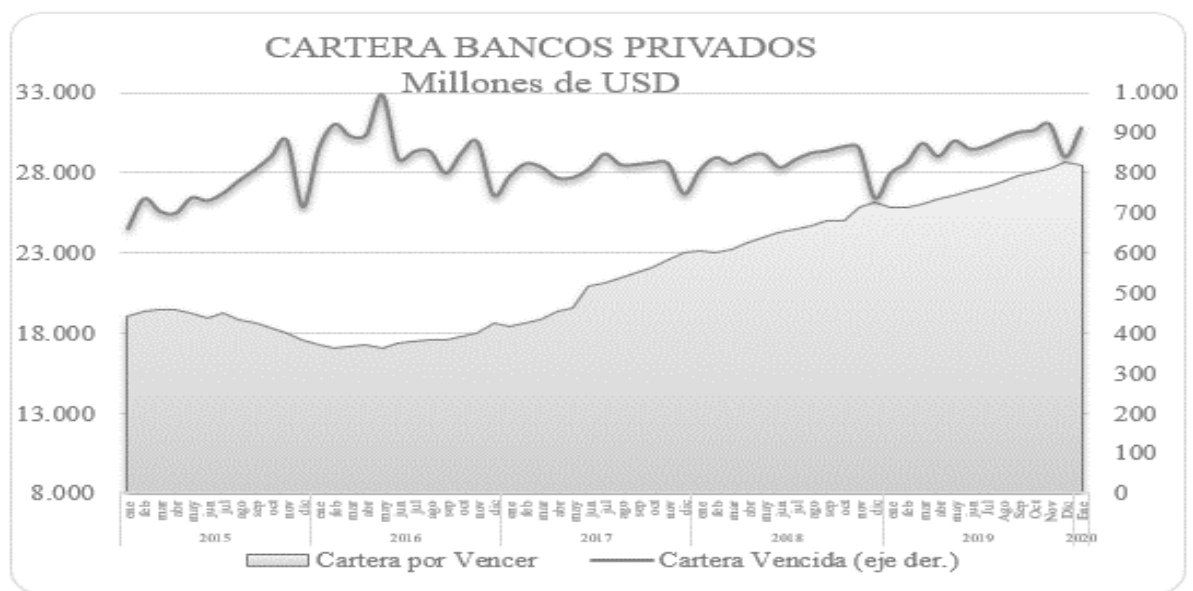

En el componente de captaciones la Banca privada durante los últimos años en lo relacionado con depósitos a la vista se ha mantenido entre los $\$ 8.000$ millones y en lo referente a depósitos a plazo, bonos del estado fluctúa entre $\$ 15.000$ y $\$ 19.000$ millones los cuales en su mayoría fue entregados por el estado a las personas jubiladas, sin embargo, hasta la actualidad existe la inconformidad de algunos de los beneficiarios ya que no pueden obtener el dinero en el tiempo establecido por la situación actual que se vive a nivel nacional y mundial (ASOBANCA, 2020).

\section{Figura 6}

Captaciones Bancos Privados

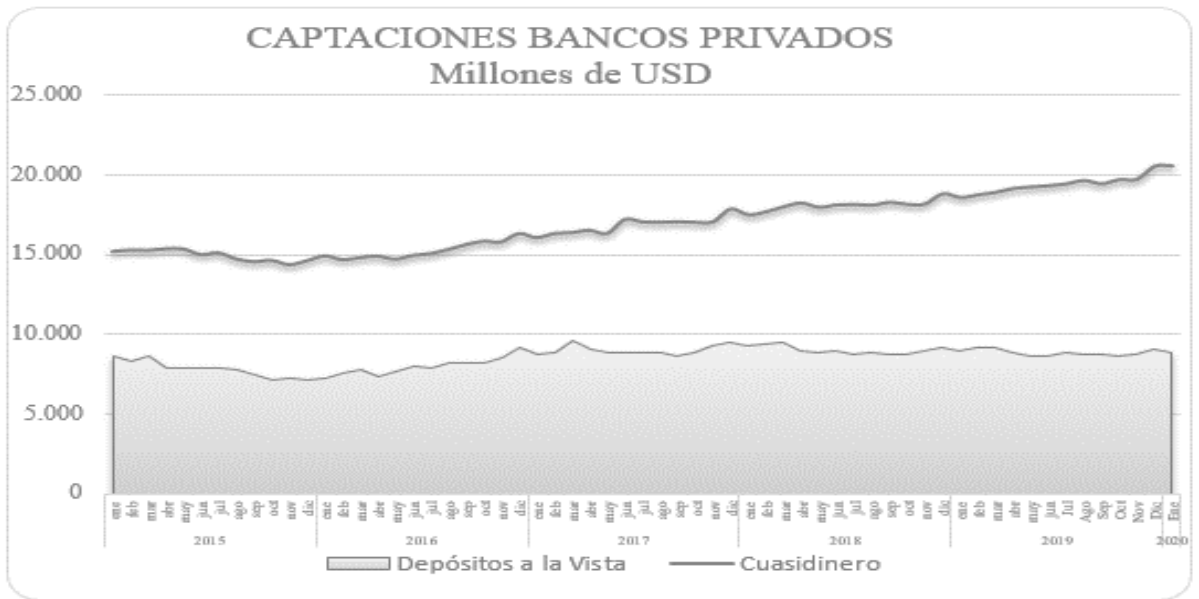

Es importante analizar la evolución de las principales cuentas de activos, pasivos, patrimonio, ingresos, gastos y resultados de la banca nacional de junio del 2019 a junio del 2020, teniendo los siguientes 
resultados presentados en el cuadro. Al finalizar el primer semestre del año 2020, se puede observar en el rubro de total de activos la banca ecuatoriana creció en $0,8 \%$ con relación al mes de mayo. De igual manera sus pasivos de manera mensual presentan un crecimiento de $0,9 \%$. Por otro lado, el saldo de los activos registra USD 44.252 millones y pasivos por USD 39.208 millones; lo cual significa un crecimiento anual de 6,2\% respectivamente. En cuanto al patrimonio alcanzó una variación anual del 10,1\%. En lo relacionado a la cuenta de ingresos se determinó una variación del $0,2 \%$, en tanto que la cuenta de gastos se incrementó en 7,4\%. Para finalizar la utilidad neta contabilizó USD 141 millones frente los USD 296 millones que se reportó en junio del 2019 con una tendencia a la baja.

Tabla 8

Principales cuentas Bancos a junio 2019 junio 2020

\begin{tabular}{lcccccc}
\hline & jun-19 & dic-19 & may-20 & jun-20 & $\begin{array}{c}\text { Variación } \\
\text { Mensual }\end{array}$ & $\begin{array}{c}\text { Variación } \\
\text { anual }\end{array}$ \\
\hline Total, activos & 41.672 & 44.583 & 43.882 & 44.252 & $0,8 \%$ & $6,2 \%$ \\
Contingentes & 12.688 & 13.387 & 13.386 & 12.991 & $-2,9 \%$ & $2,4 \%$ \\
Total, Pasivos & 36.925 & 39.536 & 38.863 & 39.208 & $0,9 \%$ & $6,2 \%$ \\
Patrimonio & 4.452 & 5.047 & 4.894 & 4.903 & $0,2 \%$ & $10,1 \%$ \\
Ingresos & 2.446 & 5.060 & 2.061 & 2.452 & & $0,2 \%$ \\
Gastos & 2.151 & 4.444 & 1.936 & 2.311 & & \\
Utilidad Neta & 296 & 616 & 126 & 141 & & $-52,2 \%$ \\
\hline
\end{tabular}

Nota. Elaborado a partir de ASOBANCA (2020).

\section{Rendimiento y contribución a la economía}

Con respecto al rendimiento y contribución a la economía por parte del sector financiero se presenta a continuación una tabla resumen de las principales cuentas de balance y los indicadores financieros tanto de las Cooperativas Segmento I y II, así como de los Bancos Privados con corte a junio 2020.

\section{Tabla 9}

Rendimiento y principales indicadores financieros a junio 2020

\begin{tabular}{|c|c|c|}
\hline Indicador Financiero & Cooperativas Segmentos I y II & Bancos \\
\hline Activos & 13.703 millones & 44.252 millones \\
\hline Cartera Bruta & 10.143 millones & 28.892 millones \\
\hline Depósitos & 10.652 millones & 33.527 millones \\
\hline Patrimonio & 1.991 millones & 4.903 millones \\
\hline Liquidez & $26.1 \%$ & $28.8 \%$ \\
\hline Solvencia & $18 \%$ & $13.9 \%$ \\
\hline Rentabilidad ROE & $5.6 \%$ & $5.7 \%$ \\
\hline
\end{tabular}




\begin{tabular}{lcc}
\hline Rentabilidad ROA & $0.8 \%$ & $0.6 \%$ \\
Cobertura & $142 \%$ & $253 \%$ \\
Eficiencia & $116 \%$ & $108 \%$ \\
Morosidad & $4.1 \%$ & $2.8 \%$ \\
Vulnerabilidad del Patrimonio & $20.2 \%$ & $16.3 \%$ \\
Intermediación Financiera & $97 \%$ & $89.8 \%$ \\
Calidad de Activos & $112 \%$ & $128 \%$ \\
Participación Crédito al Sector Productivo & $46.8 \%$ & $59.5 \%$ \\
Participación Crédito de Consumo & $53.2 \%$ & $40.5 \%$ \\
\hline
\end{tabular}

Nota. Elaborad a partir de ASOBANCA (2020).

\section{El sistema financiero y su contribución al Producto Interno Bruto}

El producto interno bruto (PIB), es una magnitud macroeconómica que expresa el valor monetario de la producción de bienes y servicios de demanda final de un país o región durante un período determinado, normalmente de un año o trimestrales. También debemos diferenciar entre PIB nominal que es el valor, a precios de mercado, de la producción de bienes y servicios finales producidos en un país durante un determinado periodo de tiempo, que suele ser un año, y el PIB real que es el valor de dicha producción a precios constantes. En la siguiente tabla encontramos la variación del PIB nominal y real desde el año 2015 al 2019 en millones de dólares y el porcentaje de crecimiento anual del PIB real. Se observa claramente que en el año 2015 el PIB real crece en un 3,9\% con relación al año anterior y en el año 2019 llega a 0,10\%.

\section{Tabla 10}

Producto Interno Bruto anual del país

\begin{tabular}{lccccc}
\hline & $\mathbf{2 0 1 5}$ & $\mathbf{2 0 1 6}$ & $\mathbf{2 0 1 7}$ & $\mathbf{2 0 1 8}$ & $\mathbf{2 0 1 9}$ \\
\hline PIB Nominal & 99290 & 99938 & 104296 & 107562 & 107436 \\
PIB Real & 70175 & 69314 & 70956 & 71871 & 71909 \\
Tasa de crecimiento anual del PIB Real & $3,90 \%$ & $-1,20 \%$ & $2,40 \%$ & $1,30 \%$ & $0,10 \%$ \\
\hline
\end{tabular}

Nota. Elaborado a partir de las Estadísticas Banco Central del Ecuador (2020).

El comportamiento del PIB nominal y real en el sector financiero se presenta en el siguiente cuadro, en el cual se puede observar que en el año 2015 tiene un crecimiento de $-0,7 \%$ y en el año 2019 tiene un crecimiento de $5 \%$ lo que nos indica la importancia de la contribución del sector en la economía ecuatoriana y como este ha ido creciendo.

\section{Tabla 11}


Producto Interno Bruto anual del sector financiero

\begin{tabular}{lccccc}
\hline & $\mathbf{2 0 1 5}$ & $\mathbf{2 0 1 6}$ & $\mathbf{2 0 1 7}$ & $\mathbf{2 0 1 8}$ & $\mathbf{2 0 1 9}$ \\
\hline PIB Nominal & $3.164,7$ & $3.072,6$ & $3.536,3$ & $3.688,1$ & $3.905,3$ \\
PIB Real & $2.227,8$ & $2.200,5$ & $2.339,3$ & $2.406,3$ & $2.526,4$ \\
Tasa de crecimiento anual del PIB Real & $-0,70 \%$ & $-1,20 \%$ & $6,30 \%$ & $2,90 \%$ & $5,00 \%$ \\
\hline
\end{tabular}

Nota. Estadísticas Banco Central del Ecuador (2020).

\section{Discusión}

El sistema financiero de la economía popular y solidaria está conformado por las cooperativas de ahorro y crédito distribuidas en los cinco segmentos de acuerdo con su valor de activos, por su tipo de relación que mantienen con sus socios y por la ubicación geográfica en zonas de alta ruralidad y pobreza estas entidades son actores claves para proveer servicios financieros a poblaciones vulnerables. La Superintendencia de Economía Popular y Solidaria a diciembre del 2019 el sector cooperativo mantenía 7,79 millones en certificados de aportación, 16.132 millones en activos y 13.784 millones de dólares en pasivos. Representando un tercio de la actividad financiera del país al concentrar el $27,2 \%$ de captaciones y el $28,6 \%$ de las colocaciones del sistema financiero privado.

El sistema financiero ecuatoriano cuya estructura está conformada por un conjunto de instituciones del sector público y privado. En lo referente a la Banca en la actualidad se cuenta con 32, de las cuales 25 corresponden al sector privado y 7 al sector público. Controladas por la Superintendencia de Bancos, que frente a la declaratoria de emergencia sanitaria en el país y el mundo, las medidas de confinamiento, la disminución de actividades productivas y económicas, entre otros factores, han requerido ejecutar medidas que generen alivio financiero a las personas, empresas, MiPymes. Es así que las IFIS en el país y la región han desempeñado un rol fundamental acatando las disposiciones de gobierno en cuanto a facilidades de pago y refinanciamiento para sus clientes. A pesar de esta situación la Banca continúa liderando el mercado financiero como lo expresa el reporte de la ASOBANCA (2020), la cual determinó que a finales de junio registró 44.252 millones en Activos, depósitos 33.527 millones, su cartera bruta registró 28.892 millones y su patrimonio 4.903 millones, lo que demuestra que a pesar de la crisis nacional y mundial el trabajo ha sido continuo en beneficio de la sociedad.

La Banca si bien es cierto ha sufrido una serie de cambios en los últimos años como consecuencias de las crisis económicas en los diferentes periodos, sin embargo, busca las estrategias para generar mayor valor a las entidades financieras, mejorar la calidad de atención tanto al cliente interno y externo, los aspectos referentes a la medición y gestión del riesgo, de la mano de tecnología de punta y el contar con el 
elemento humano idóneo para el desenvolvimiento de sus actividades. Según reporte de la revista EKOS del mes de junio del 2020, determinó cuales son los Bancos líderes en servicios financieros y los que van a la vanguardia en tecnología, experiencia y servicio al cliente ocupando el primer lugar el Banco del Pichincha. Este segmento determinó un crecimiento de $5 \%$ de su total de activos, un 1,9\% en sus depósitos y un $11 \%$ en su cartera de créditos. Manteniendo una eficiencia del $4,78 \%$.

Es indiscutible el compromiso de la Banca para ofrecer productos y servicios de la mano de nuevas tecnologías, que aporten al crecimiento de la población con el financiamiento de proyectos productivos de inversión que aporten al cambio de la matriz productiva, proveer de servicios que permitan una mayor bancarización e inclusión financiera de la sociedad ecuatoriana, enmarcado en una estructura financiera adecuada y acorde a las normas establecidas por los organismos de control.

Es importante considerar el efecto COVID en el sistema financiero nacional, según datos de información publicada en revista EKOS del mes de agosto del 2020 al cierre del primer semestre se observa que el sistema contó con la confianza de sus socios que mantuvieron los niveles de depósitos. El diferimiento de los créditos, ordenado por el Gobierno entre marzo y junio, afectó los procesos normales de recuperación y reinversión de la cartera de crédito. En el caso del sistema cooperativo, el 55\% de los créditos se difirieron para el segundo semestre y no está claro cómo ello afectará a los niveles de morosidad y provisiones del sistema. Se espera que la calidad de la cartera se deteriore en las nueve provincias más afectadas por la pandemia (Guayas, Pichincha, Manabí, Santo Domingo, Esmeraldas, El Oro, Los Ríos, Azuay y Loja), en especial en aquellas actividades que se han paralizado de forma importante como es el caso del sector de la Hospitalidad, Construcción y Comercio.

Otro efecto es la desaceleración en la cartera de crédito, debido al impacto del COVID 19 en la economía nacional, factor que produce la reducción en la intermediación financiera causando un efecto negativo en los resultados, varias instituciones reportarán pérdidas o verán reducidos sus márgenes como se observó en la variación de la Utilidad Neta del -35,9\% en el sector cooperativo y del -52.2\% en la banca con respecto a junio 2019.

La tarea de la Superintendencia de Bancos, la Superintendencia de la Economía Popular y Solidaria y de la Junta de Regulación y Política Monetaria resulta clave ya que se debe normar, controlar y evitar que el incremento de la morosidad y provisiones, una vez que termine el periodo de diferimiento, afecte el patrimonio de las entidades. El sistema financiero se mantiene sólido y líquido, sin embargo, los índices de rentabilidad han disminuido por la situación antes descrita, debiendo ser monitoreado y vigilado para evitar un deterioro por los riesgos del entorno. Pese a esto la responsabilidad y oportunidad que tienen 
las instituciones, es la de apoyar la recuperación y reactivación de las actividades productivas que necesitan liquidez y servicios financieros para encender nuevamente el motor de la economíax

\section{Conclusión}

Se puede concluir que existen características diferenciadoras profundas desde los aspectos filosófico y social entre estos dos representantes del sistema financiero nacional, destacándose que las Cooperativas de Ahorro y Crédito tienen una fuerte presencia en el ámbito social regida por principios y valores; en tanto que el sector bancario prevalece como número uno en cuanto a cobertura y movilización de capitales. De esta forma el sistema financiero popular y solidario muestra un dinamismo y crecimiento importante reflejado en sus niveles de activos, cartera y depósitos, convirtiéndose en las entidades de mayor crecimiento en el sector financiero privado. Sin embargo, dada su heterogeneidad marcada por la diferencia de tamaño, tecnología, gobierno corporativo y patrimonio, entre las del Segmento 1 respecto al resto de entidades que lo conforman, es necesario una supervisión activa para reducir los niveles de exposición al riesgo en especial de las instituciones más pequeñas.

La Banca Ecuatoriana requiere de igual manera que se generen estrategias de mejora en los diferentes procesos que permitan recuperar la confianza de los clientes. Por lo cual, es de vital importancia el analizar de manera permanente los riesgos, con el propósito de aplicar las medidas de protección que incrementen la capacidad, física, técnica, personal, organizativa, minimizando así las vulnerabilidades al que están expuestas y las amenazas que diariamente se puede presentar.

\section{Referencias}

Akenten, W., Ayertey, A., y Andoh, R. (2009). Credit Unions in Developing Economies: Membership Benefits. http://dx.doi.org/10.2139/ssrn.3346334

Asamblea Nacional Constituyente. (2008). Constitución de la Republica de Ecuador. https://educacion.gob.ec/wp-content/uploads/downloads/2012/08/Constitucion.pdf

Asamblea Nacional de la República del Ecuador. (2014). Registro oficial No 332, segundo suplemento. http://www.pge.gob.ec/documents/Transparencia/antilavado/REGISTROOFICIAL332.pdf:

Asamblea Nacional del Ecuador. (2011). Ley Orgánica de Economía Popular y Solidaria. https://www.seps.gob.ec/documents/20181/25522/LEY\%20ORGANICA\%20DE\%20ECONOMIA \%20POPULAR\%20Y\%20SOLIDARIA\%20actualizada\%20noviembre\%202018.pdf/66b23eef8b87-4e3a-b0ba-194c2017e69a 
Asamblea Nacional del Ecuador. (2014). Código Orgánico Monetario y Financiero. http://www.pge.gob.ec/documents/Transparencia/antilavado/REGISTROOFICIAL332.pdf

Asociación de Bancos privados [ASOBANCA]. (2020). Evolución de la Banca Privada Ecuatoriana. 11-13. http://investiga.ide.edu.ec/images/pdfs/2016junio/Perspectiva Junio 2016.11-13.protected.pdf.

Banco Central del Ecuador. [BCE]. (2012). ABC del BCE. Quito: Departamento publicaciones económicas. https://contenido.bce.fin.ec/documentos/PublicacionesNotas/Catalogo/Memoria/2008/abcweb.pdf

Banco Central del Ecuador. [BCE]. (2020, 15 de junio). Estudios Económicos. https://estudioseconomicos.bce.fin.ec/index.php/RevistaCE/

Banco Central del Ecuador. [BCE]. (2021, 20 de julio). Conozca el Banco Central. https://www.bce.fin.ec/index.php/funciones-del-banco-central

Carvajal, A., \& Zuleta, H. (1997). Desarrollo del sistema financiero y crecimiento económico. Borradores de Economía, 67. https://repositorio.banrep.gov.co/handle/20.500.12134/5083

Castelló, E., y Trías, C. (2015). Cooperatives and Savings Banks in the European Economic Area: A Strategic Vision for the Future. Boletín de Estudios Económicos, LXX, 555-570. Retrieved from https://search-proquest-com.vpn.ucacue.edu.ec/docview/1764137133?accountid=61870

Die Genossenshaften. [DGRV]. (2019). Datos y Ranking de Cooperativas de Ahorro y Crédito en América Latina el Caribe. https://collaboration.worldbank.org/content/usergenerated/asi/cloud/attachments/sites/collaboratio n-for-development/en/groups/cooperative-financialinstitutions/documents/jcr:content/content/primary/blog/data_and_rankingofiaeJ/DGRVCifrasRankingCACALC_2019\%20final.pdf

Redacción Negocios, (2019, 15 de junio). Las cooperativas de ahorro y crédito crecieron 132\% en 7 años. El Comercio,6

Espinosa, R. (2000). La crisis económica financiera ecuatoriana de finales de siglo y la dolarización. Universidad Andina Simón Bolívar, 1-9. http://memoriacrisisbancaria.com/www/articulos/Roque_Espinosa_Crisis_bancaria.pdf

Hannan, T. (2002). The impact of credit unions on the rates offered for retail deposits by banks and thrift institutions. https://www.federalreserve.gov/pubs/feds/2003/200306/200306pap.pdf

Hernández Torres, N. (2017). La evolución del sistema Bancario. Tesis de Grado en Contabilidad y Finanzas, Universidad de la Laguna. https://riull.ull.es/xmlui/bitstream/handle/915/7510/La\%20Evolucion\%20del\%20Sistema\%20Banc ario.pdf?sequence $=1$ 
Ley Orgánica de Instituciones del Sistema Financiero. (2014). Código orgánico monetario y financiero capítulo content/uploads/downloads/2020/12/codigo_organico_monetario_financiero2.pdf

López del Paso, R. (2011). El origen del término Bancarrota. Extoikos, 2, p.141-142. https://dialnet.unirioja.es/descarga/articulo/4019428.pdf

Martínez, H., Rivas, E., Rositas, J., \& Treviño, E. (2015). El conocimiento Adecuado de los instrumentos financieros y las crisis del 2008: Impacto de las decisiones de la alta dirección en México. Vinculatégica EFAN 1(1), 1113-1134. http://eprints.uanl.mx/17240/1/59.pdf

McKillop, D., French, D., Quinn, B., Sobiech, A. L., y Wilson, J. O. S. (2020a). Cooperative financial institutions: A review of the literature. International Review of Financial Analysis, 71(December 2019). https://doi.org/10.1016/j.irfa.2020.101520

Muñoz, E. C., \& Pintó, C. T. (2015). Las cooperativas de crédito y cajas de ahorro en el espacio económico europeo: una visión estratégica de futuro $\left(^{*}\right) /$ Cooperatives and savings banks in the european economic area: a strategic vision for the future. Boletín de Estudios Económicos, 70(216), 555569. https://www-proquest-com.vpn.ucacue.edu.ec/scholarly-journals/las-cooperativas-de-créditoy-cajas-ahorro-en-el/docview/1764137133/se-2?accountid=61870

Muñoz, E. C., \& Pintó, C. T. (2015). Las cooperativas de crédito y cajas de ahorro en el espacio económico europeo: una visión estratégica de futuro $\left(^{*}\right) /$ Cooperatives and savings banks in the european economic area: a strategic vision for the future. Boletín de Estudios Económicos, 70(216), 555569. https://www-proquest-com.vpn.ucacue.edu.ec/scholarly-journals/las-cooperativas-de-créditoy-cajas-ahorro-en-el/docview/1764137133/se-2?accountid=61870

Romero, P. (2007). Crisis bancaria en Ecuador: causas y posibles soluciones. Observatorio de la Economía Latinoamericana, http://memoriacrisisbancaria.com/www/articulos/Crisis_bancaria_Ecuador_causas_soluciones.pd f

Segui, E., Mateos, A., Mari, S., \& Zamorano, M. (2011). Factores determinantes en la predicción del fracaso empresarial en cooperativas: Un análisis Delphi. Centro de Investigación en Gestión de Empresas Universitat Politécnica de València. http://www.aeca1.org/pub/on_line/comunicaciones_xvicongresoaeca/cd/169i.pdf

Superintendencia de Economía popular y solidaria. [ SEPS]. (2018). Ley orgánica de economía popular y solidaria.

https://www.seps.gob.ec/documents/20181/25522/LEY\%20ORGANICA\%20DE\%20ECONOMIA \%20POPULAR\%20Y\%20SOLIDARIA\%20actualizada\%20noviembre\%202018.pdf/66b23eef8b87-4e3a-b0ba-194c2017e69a

Superintendencia de Bancos. [SIB]. (2020). Estadísticas, información financiera, SIB: www.sbs.gob.ec 
Superintendencia de Bancos. [SIB] (2020). Portal del Usuario Financiero. Educación Financiera. https://estadisticas.superbancos.gob.ec/portalestadistico/efinanciera/?page_id=38

Superintendencia de Economía Popular y Solidaria. (2018). Informe de Rendición de Cuentas 2018. https://www.seps.gob.ec/rendicion-de-cuentas?rendicion-de-cuentas-2018

Superintendencia de Economía Popular y Solidaria. (2019). Conoce que es la Economía Popular y Solidaria (EPS) - SEPS. http://www.seps.gob.ec/interna?conoce-la-eps

Universidad Nacional del Ecuador. [UNAE]. (2019) El Sistema Financiero Módulo 2, Programa de Aprendizaje cooperativo para Jóvenes, recuperado de: Jardín Azuayo, https://issuu.com/jardinazuayo/docs/modulo_2_25e655ddfd5bfb

Tristán, P., Guevara, M., \& Cortéz, K. (2019). Análisis comparativo entre criptomonedas y el dinero $\begin{array}{lll}\text { fiduciario. } & \text { 8inculatégica } & \text { 80.89. }\end{array}$ http://www.web.facpya.uanl.mx/Vinculategica/vinculategica_5/8\%20TRISTAN_GUEVARA_CORT EZ.pdf

Terrones, C. (2013). Clasificación de la banca comercial peruana: Un análisis de clúster jerárquico. Obtenido de http://www.sbs.gob.pe/Portals/0/jer/ddt_ano2013/DT_04_2013.pdf

Turner, W. (1996). Credit unions and banks: turning problems into opportunities in personal banking, International Journal of Bank Marketing, $14 . \quad$ (1), 30-40. https://www.emerald.com/insight/content/doi/10.1108/02652329610105305/full/html.

\section{Contribución de los autores}

JCM: Conceptualización, análisis formal, metodología, investigación, redacción: revisión y edición.

ETC: Conceptualización, análisis formal, metodología, investigación, redacción: borrador original.

GCS: Conceptualización, análisis formal, metodología, investigación, redacción: borrador original.

JOP: Conceptualización, análisis formal, metodología, investigación, redacción: borrador original.

\section{Fuentes de financiamiento}

La investigación fue realizada con recursos propios.

\section{Conflictos de interés}

Los autores declaran no tener conflictos de interés.

\section{Correspondencia}

jordonezp@ucacue.edu.ec 